THE JOURNAL OF TRANSPORT AND LAND USE http://jtlu.org

vOL. 6, NO. 3 [2013] pp. 61-74 doi: 10.5198/jtlu.v6i3.350

\title{
A multidimensional decisions modeling framework for built space supply
}

\author{
Bilal Farooq ${ }^{a}$ \\ École Polytechnique Montréal
}

\author{
Eric J. Miller \\ University of Toronto
}

\author{
Murtaza Haider \\ Ryerson University
}

\begin{abstract}
The spatial and temporal distribution of built space supply plays an important role in shaping urban form and thus the general travel pattern in an urban area. Within an integrated framework, we are interested in modeling the decisions of a builder in terms of when, where, what type, and how much built space to build. We present a multidimensional discrete-continuous model formulation for the built space supply decisions that are based on expected profit maximization. The framework is applied to estimate a model for supply of new office space in the greater Toronto area (GTA) for the 1986 to 2006 period. To our knowledge, this work is the first that models the where, when, how much, and what type of office space to build in a single econometric framework at a fairly disaggregate spatial zoning system. The results indicate risk taker behavior on the builders' part, while market conditions and supply of resources (labor, construction cost, etc.) are also found to be important factors in decision making.
\end{abstract}

\section{Introduction}

Understanding the factors that affect built space location/ relocation decisions plays an extremely important role in our greater understanding of travel behavior in urban areas. Since activity location, especially residential and work related, strongly influences the spatial distribution of morning and afternoon peak-period travel, where households and firms choose to locate greatly influences short-term, individual-level decisions, such as mode of transportation, and long-term householdlevel decisions, such as residential/work relocation. Conditions in the built space market affect households/firms' location and relocation decisions, and hence influence the general travel patterns in an urban area. Supply decisions constitute an important dimension of decision making in the context of built space markets. Builders in the built space market respond to market conditions, availability of land and other resources (e.g., capital, construction material, etc.), and regional economic conditions by adjusting the supply of different categories of built space. In the case of brownfield redevelopment (De Sousa, 2002), builders transform an existing built space into a new space of the same category or a different category. In terms of the total inventory of built space in the urban area, due to these rebuild decisions by the builders, the stock of one category of built space could decrease and the stock of another might increase. Brownfield development usually requires changes in zoning bylaws that are related to the land parcel. In the outer limits of an urban area, landowners sell land to developers. In the context of the greater Toronto area (GTA), most of the time, developers will buy cheap agricultural land surrounding the city many years in advance and keep it until the local municipality designates it for development. Developers then help shape the final zoning of the land and develop it into parcels. Builders, based on zoning and expected demand, build different categories of built space on these parcels. A developer and a builder can be the same or different agents. An analysis of the InfoCanada ${ }^{2}$ dataset for businesses operating in the GTA in 2006 shows that there are fewer than 500 builders operating in the GTA. Buzzelli and Harris (2003) suggest that the number of active developers is even less than the number of builders. Developers usually work with the same builders, and in very few cases, the developer of land also builds the space. This supports the argument that the built space supply market in the GTA is a well-connected oligopoly. The analysis of the InfoCanada dataset on builders shows that in 2006 the sales volume of $13 \%$ of the builders was less than $\$ 1$ million $(\mathrm{C})^{3}, 70 \%$ of the builders had sales between $\$ 1$ million and $\$ 10$ million, and $17 \%$ had sales of more than $\$ 10$ million. The building industry is thus dominated numerically by small- to medium-sized builders, but at the same time there is a significant presence of heavy-weight players in the industry. Buzzelli and Harris (2003) similarly report that the building industry in Ontario

\footnotetext{
a bilal.farooq@polymtl.ca

${ }^{1}$ Built space is a generic term used throughout this paper to represent various types of spaces in an urban area that have a physical structure and associated monetary value; it can be identified as individual quasi-unique units (based on their attributes and location) and provide opportunities for various activities. These spaces include: dwelling units, office spaces, retail spaces, industrial spaces, etc.

${ }^{2}$ InfoCanada is a marketing consultant firm that conducts surveys related to business firms in various major cities of the world, including Toronto.

${ }^{3}$ All the monetary values are in Canadian dollars.
} 
has a high number of small- to medium-sized builders. The total volume of the sales from the building industry in 2006 was approximately $\$ 5$ billion, with small to medium builders contributing $\$ 800$ million of this total. The large-sized builders contributed $\$ 4.2$ billion, which is more than five times that contributed by the small- and medium-sized ( $83 \%$ numerically) builders. This shows that the large-sized builders play a dominating role in the building industry. Another interesting fact about the building industry is that about $95 \%$ of the builders have fewer than 25 employees. This is because builders do not perform the construction job in-house. Instead, they heavily rely on contractors and subcontractors to actually do the job for them, and the builders' employees are usually only managing the project. Buzzelli and Harris (2003) reported that this relation between the builders and contractors is spatially localized and long term.

The building project has various identifiable stages (Somerville, 2001) (Figure 1). In the first stage, a builder applies for a permit to construct a certain quantity of built space, seeks any required zoning changes, and acquires financial backing. Once approved, the builder may start the construction of the entire or some quantity of the built space it is permitted to build. The time to start the construction can vary, depending on market and regional economic conditions, but the latest time to start is dictated by the terms and conditions of the loans. The completion time of the projects can also vary depending on these conditions. The introduction of space within the market can vary both in terms of time and quantity. Moreover, the whole project construction process can vary for different categories of built space.

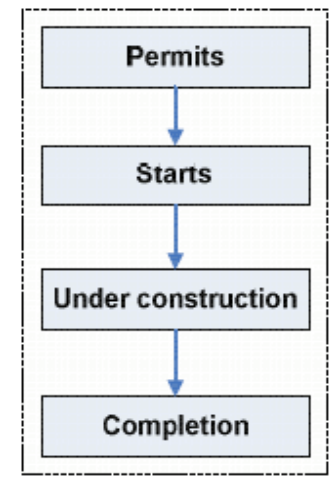

Figure 1: Various stages of construction

Supply of new built space is a very complex process in which there are markets (land, development, and building), various stages, different types/levels of finished product (land, developed parcels, and built space), and various types of de- cision-making agents (landowners, developers, and builders) (Figure 2). One approach to deal with this complexity is the grid cell concept (Waddell et al., 2008; Hunt et al., 2007). The urban area is divided into fixed grid cells that act as evolving cellular automata. It is a very rough abstraction of the developer and builder agents. The grid cell maintains its own inventory and decides on the built space supply decisions (land is the decision maker). It seems to be an over simplification of the process. We think that the different markets, finished products, and agents should be identified separately with inter- and intratype interaction. Moreover, the parcels evolve by merging and splitting (especially in the case of brownfield redevelopment). The concept of a fixed dimension grid cell cannot represent this evolution. Martinez and Roy (2004) within the equilibrium framework modeled the supply process as a chain of market processes in which landowners, developers, and builders interact. Their modeling approach is a better representation of the various markets, finished products, and agents involved in the built space supply. But the strong equilibrium assumption fails to capture the complex interactions occurring among the various agents within these markets.

Realizing the highly complex nature of the supply process that results from the behavior and interactions of the involved agents, in this paper, we only focus on the decision-making behavior of one agent, the builder. In the context of new built space supply, a single builder is faced with various dimensions of decisions. The builder has to decide when, where, what type, and how much to build so that it can maximize its expected profit. In this context, we assume that the builder:

- Has access to financial and other resources required to build the amount of space it decides to build

- Can acquire land parcels anywhere in the urban area

- Can get a permit for the construction of the quantity it desires to build

- Has a fixed time of construction

- Introduces at once the entire quantity of built space to the built space market at the end of the project

- Is an expected profit maximizer and computes expected profits at the start of the project by speculating about future revenues from sale, rent, and lease and various costs associated with the project

Under these assumptions we propose a novel approach that explicitly ties time, location, quality, and quantity decisions for new built space supply together into a single dynamic framework that is based on expected profit maximization. As an application, we then estimate a model for new office space development at a fairly disaggregate spatial resolution. We treat 
the problem of building new built space as a situation in which a builder as a decision maker is faced with the decision of selection of a choice bundle and the associated quantities, while optimizing expected profits. By doing so, we were able to incorporate not only the relation between various decision dimensions but also to capture the behavior of the decision maker (builder) and the influence of changing submarket conditions and regional economy on the decision making. The rest of the paper is organized as follows: We first present the model formulation and then the application of the model to the new office space supply model. At the end of this paper, we present concluding discussion and future directions.

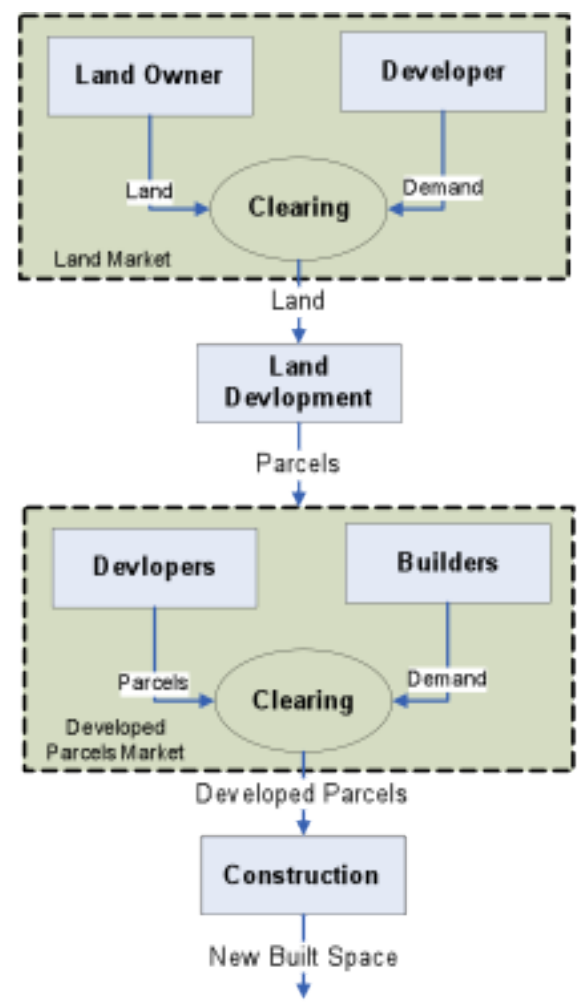

Figure 2: Various markets and agents involved in the built apace supply

\section{$2 \quad$ Model of new built space supply}

Here we present the theoretical formulation of the model for new built space supply that models the multidimensional decisions of when, where, what type, and how much to build in a single consistent modeling framework. The decision makers are a set of building construction firms (builders) that are active in the urban area at certain time t. They are faced with the decision of choosing the quantity of different types of built space to be built and the location where to build them. It is assumed that builders make these decisions so as to maximize their expected profits. Profit is determined by the difference between expected revenue and cost. There are various large-scale demand models available in the choice modeling literature that model the choice of a discrete bundle of goods (e.g., types of activities in which to engage in) and an associated continuous quantity (e.g., how much time to allocate to each activity) (Bhat, 2005, 2008; Habib et al., 2008; Habib and Miller, 2009; Kim et al. 2002). These models predominantly use the well-known random utility modeling (RUM) framework that assumes that the consumer is a utility maximizer. In terms of mathematical model formulation, the assumption of profit maximization by the producer (specifically, builders in this case) is analogous to the utility maximization assumption in these large-scale demand models. Moreover, profit from manufacturing a product is a more quantifiable concept than utility. Thus we can pose the problem of expected profit maximization in the same way RUM does for the utility maximization of consumers faced with choice bundle selection and the associated quantities. This lets us use the same construct of optimization conditions (Kuhn-Tucker conditions) that in recent years has frequently been used in large-scale demand models.

\section{$2.1 \quad$ Theoretical Framework}

The expected profit (П) of a building construction firm, from $\mathrm{N}$ differentiated products that it can decide to build at certain decision point $\mathrm{t}$ can be represented by:

$$
\Pi=\sum_{i=1}^{N} \frac{\gamma_{i}}{\alpha_{i}}\left\{\left(f^{r}\left(X_{i}^{r}\right)-f^{c}\left(X_{i}^{c}\right)\right)^{\vartheta}\left(\left(\frac{q_{i}}{\gamma_{i}}+1\right)^{\alpha_{i}}-1\right)\right\}+f^{z}(z)
$$

Where:

$f^{r}\left(X_{i}^{r}\right)$ is a hedonic function that represents the expected unit revenue from selling product $\mathrm{i}$

$X_{i}^{r}$ is a vector of variables related to product attributes, location features, and built space market conditions that influence the revenue

$f^{c}\left(X_{i}^{c}\right)$ is a hedonic function that represents the expected unit cost in building product $\mathrm{i}$

$X_{i}^{c}$ is a vector of variables related to product attributes, location, state of regional economy, and conditions in various associated markets (labor, material, etc.) that influence the cost

$q_{i}$ is the quantity of product $i$ that is decided to be built

The formulation here treats the share of profit from individual type of floor space $i$ in the same manner as Bhat (2005) and Kim et al. (2002) treat the share of individual choices in their utility function for large-scale demand systems. The translation parameter $\gamma_{i}$ makes sure that there is a possibility of zero production of any given type of built space. Its value is greater or equal to zero $\left(\gamma_{i} \geq 0\right)$ so as to make sure that the indifference curves touch the horizontal axis with a finite slope (Bhat, 
2008). The parameter $\alpha_{i}$ is a scale parameter that adjusts the marginal profit with respect to the associated quantity of built space. Here, 9 represents the risk behavior of the builder and the structure of the space market in the region. In the simplest case, $\vartheta$ could be a constant parameter, but in a more elaborate case, it could be parameterized based on a combination of the builder's and the market's characteristics. The value of $\vartheta$ greater than one would mean that the builders inflate the expected revenue thus showing a greater risk-taker attitude, while a lower than one value would represent a risk-avoidance attitude due to deflation of the expected revenue. Bhat (2008) performed a comprehensive analysis of the influence of different values of $\gamma_{i}$ and $\alpha_{i}$ on the indifference curves for the utility of the goods consumed. Similar analysis is needed for the formulation suggested in equation [1] for the profit.

\subsubsection{Concept of Hicksian/composite product}

As the builder has other options of investment, besides the set of built space types that we are modeling, we introduce the concept of a Hicksian/composite product in our general formulation, $f^{z}(z)$. Here, $\mathrm{z}$ is in the terms of built space units (square foot in the case office space). The value of which comes from the difference between the maximum that can be built and the sum of all the quantities $\left(q^{*}\right)$ of different types of built space that were built by the builder in the time step $t$. If the conditions are extremely favorable, the builder would like to build as much as possible. The only limiting factor will be the technological or zonal constraints, as builders can only build to a certain extent with the current technology in a given time step. On the other hand, if the conditions are not highly favorable, the builder will carefully select the supply levels to an extent so that profit is maximized and loss avoided by overbuilding. Profit from the Hicksian product in equation [1] represents the expected loss that is avoided at a given interest rate at the decision time by not building the built space that could have been built under the technological/zoning constraint. For this, we use a separate profit generation function similar to the one used by von Haefen and Phaneuf (2004) and Habib and Miller (2009) for the composite activity. If we assume that the revenue and cost functions are linear in parameters and the modeler's inability to perfectly observe the builder's expected profit is represented by the error term $\varepsilon_{1}$, then equation [1] can be rewritten as:

$\Pi=\sum_{i=1}^{N} \frac{\gamma_{i}}{\alpha_{i}}\left\{\left(\beta_{i}^{r} X_{i}^{r}-\beta_{i}^{c} X_{i}^{c}\right)^{\vartheta} e^{\vartheta \varepsilon_{i}}\left(\left(\frac{q_{i}}{\gamma_{i}}+1\right)^{\alpha_{i}}-1\right)\right\}+\frac{1}{1-e^{\rho}} z^{\left(1-e^{\rho}\right)}$

The form of the profit function for composite product here guarantees a positive profit from a nonzero composite product (Habib and Miller, 2009).

\subsection{Estimation Problem}

Using equation [2], our optimization problem can be defined as:

Maximize

$\Pi=\sum_{i=1}^{N} \frac{\gamma_{i}}{\alpha_{i}}\left\{\left(\beta_{i}^{r} X_{i}^{r}-\beta_{i}^{c} X_{i}^{c}\right)^{\vartheta} e^{\vartheta \varepsilon_{i}}\left(\left(\frac{q_{i}}{\gamma_{i}}+1\right)^{\alpha_{i}}-1\right)\right\}+\frac{1}{1-e^{\rho}} Z^{\left(1-e^{\rho}\right)}$

Subject to
\[ \begin{array}{l}\sum_{i=1}^{N} q_{i}+z=K_{T} \\ \quad q_{i} \geq 0 i=1,2, \ldots, \mathrm{N} \\ Z>0\end{array} \]

$K_{T}$ is the maximum possible space that can be built in the time interval under zoning and technological constraints.

The Lagrangian function for the problem in [3] becomes:

$$
\begin{aligned}
\mathcal{L}=\left[\sum_{i=1}^{N} \frac{\gamma_{i}}{\alpha_{i}}\left\{\left(\beta_{i}^{r} X_{i}^{r}-\beta_{i}^{c} X_{i}^{c}\right)^{\vartheta} e^{\vartheta \varepsilon_{i}}\left(\left(\frac{q_{i}}{\gamma_{i}}+1\right)^{\alpha_{i}}-1\right)\right\}+\frac{1}{1-e^{\rho}} z^{\left(1-e^{\rho}\right)}\right] \\
-\lambda\left[\sum_{i=1}^{N} q_{i}+z-K_{T}\right] \\
\lambda=\text { Lagrangian multiplier }
\end{aligned}
$$

The Khun-Tucker (KT) first order conditions for optimal allocations here will be:

$$
\begin{aligned}
& \frac{\partial \Pi}{\partial q_{i}}-\lambda \leq 0 \quad \text { for } i=1,2, \ldots \mathrm{n} \\
& \& \\
& \frac{\partial \Pi}{\partial z}-\lambda \geq 0
\end{aligned}
$$

Condition in [5a] ensures that, for the selected levels of the product bundle, any further increase in the quantity of product $i$ will have no further positive effect on the total profit. Whereas, [5b] ensures that the quantity of the composite product (not investing) is at the level where it has no negative effect on the profit. These conditions help in identifying the solution in the estimation process.

From $[5 a]$ and $[5 b]$

$$
\begin{array}{lc}
\frac{\partial \Pi}{\partial q_{i}} \leq \frac{\partial \Pi}{\partial z} \quad \text { for } i=1,2, \ldots \mathrm{n} \\
\left(\beta_{i}^{r} X_{i}^{r}-\beta_{i}^{c} X_{i}^{c}\right)^{\vartheta} e^{\vartheta \varepsilon_{i}}\left(\frac{q_{i}}{\gamma_{i}}+1\right)^{\left(\alpha_{i}-1\right)} \leq z^{-e^{\rho}}
\end{array}
$$


We can show that $\frac{\partial^{2} \Pi}{\partial q_{i} \partial \varepsilon_{i}}$ is a $i \mathrm{x} i$ non-singular matrix and $\frac{\partial^{2} \Pi}{\partial z \partial \varepsilon_{i}}$

is a zero valued vector. Thus using explicit function theorem

(Jittorntrum, 1978), we can express that error term as:

$$
\varepsilon_{i} \leq g_{i}\left(\beta_{i}^{r}, X_{i}^{r}, \beta_{i}^{c}, X_{i}^{c}, q_{i}, \vartheta, \gamma_{i}, \alpha_{i}, \rho\right)
$$

$\varepsilon_{i} \leq \frac{1}{\vartheta}\left[\left(1-\alpha_{i}\right) \log \left(\frac{q_{i}}{\gamma_{i}}+1\right)-\vartheta \log \left(\beta_{i}^{r} X_{i}^{r}-\beta_{i}^{c} X_{i}^{c}\right)-e^{\rho} \log \left(K_{T}-\sum_{j=1}^{n} q_{j}\right)\right], \forall i$

\subsection{Econometric model structure}

If the joint probability density function $\mathrm{f}\left(\varepsilon_{1}, \varepsilon_{2}, \ldots, \varepsilon_{\mathrm{n}}\right.$ of the error terms is known, then the probability associated with the quantities of a certain bundle of product that is selected by the building firm for construction is given by:

${ }^{\mathrm{P}}(Q)=\int_{-\infty}^{g_{m+1}} \ldots \int_{-\infty}^{g_{n}} f\left(g_{1}, g_{2}, \ldots g_{m}, \varepsilon_{m+1}, \ldots, \varepsilon_{n}\right)|J| d \varepsilon_{m+1} \ldots d \varepsilon$

\section{Where}

$\mathrm{Q}=\left[\mathrm{q} 1^{*}, \mathrm{q} 2^{*}, \ldots, \mathrm{qm} \mathrm{m}^{*}, 0,0 \ldots 0\right]$ is the vector of quantities of each type selected by the builder to build.

$|J|$ is the determinant of $m \times m$ Jacobian matrix, whose individual elements are defined by:

$\partial \varepsilon_{i} / \partial q_{i}$ (Kim et al., 2002; Bhat, 2005; and Habib and Miller, 2009).

$|J|=\prod_{i=1}^{N} \frac{1}{\vartheta}\left[\frac{\left(1-\alpha_{i}\right)}{\left(q_{i}+\gamma_{i}\right)}+\frac{e^{\rho}}{\left(K_{T}+q_{i}\right)}\right]$

Most of the discrete-continuous large-scale demand models including (Bhat, 2005, 2008; Habib and Miller, 2008, 2009; Habib, 2009; Pinjari and Bhat, 2009), have assumed the error terms to be independently and identically distributed (IID) with Type I extreme value distribution. This assumption simplifies [8] and gives a closed form solution for the calculation of choice probabilities. The estimation of model parameters also becomes computationally manageable in cases where the size of the choice set is large. However, we think that this assumption is not valid in the case of new built space. Most of the time, the types of space built by the builder are highly correlated to each other. Builders are localized in terms of their operations (Buzzelli and Harris, 2003). Moreover, builders and their associated contractors/subcontractors typically specialize in building specific types of space. The builder that builds detached dwellings is more likely to build semi-detached and attached dwellings than high-rise apartments. The location case is similar: A zone (business node) that primarily has Type-A office space (BOMA, 2009) will unlikely get an inferior, Type-C, office space built. A more appropriate assumption, therefore, is that the error terms are jointly normally distributed with a mean of zero and covariance matrix $\Omega$. Hence:
$P(Q)=\frac{1}{(2 \pi)^{n / 2}|\Omega|^{1 / 2}} \int_{-\infty}^{+\infty} \cdots \int_{-\infty}^{+\infty} \int_{-\infty}^{g_{m+1}} \cdots \int_{-\infty}^{g_{n}} \exp \left(-\frac{1}{2} \mathrm{E}^{\prime} \Omega^{-1} \mathrm{E}\right)|J| d \varepsilon_{1} \ldots d \varepsilon_{n} \quad[10]$

Where $\#=\left[\varepsilon_{1}, \ldots, \varepsilon_{\mathrm{m}}, \varepsilon_{\mathrm{m}+1} \ldots \varepsilon_{\mathrm{n}}\right]$

Equation [10] involves computing an $(\mathrm{n}-\mathrm{m})$ dimensional integral of the function that will have a high computational cost associated with large choice sets. In the case of built space, however, the builder is faced with very few choices (e.g., three in the case of office space and four to five in the case of housing). Thus the evaluation of [10] remains computationally viable.

The resulting likelihood function from [10] for all the builders thus becomes:

$$
\begin{aligned}
& L\left(Q \mid \beta_{i}^{r}, \beta_{i}^{c}, \vartheta, \gamma_{i}, \alpha_{i}, \rho, \Omega\right)= \\
& \quad \prod_{b=1}^{B} \frac{1}{(2 \pi)^{n / 2}|\Omega|^{1 / 2}} \int_{-\infty}^{+\infty} \cdots \int_{-\infty}^{+\infty} \int_{-\infty}^{g_{k+1}} \ldots \int_{-\infty}^{g_{n}} \exp \left(-\frac{1}{2} \mathrm{E}^{\prime} \Omega^{-1} \mathrm{E}\right)|J| d \varepsilon_{1} \ldots d \varepsilon_{n}
\end{aligned}
$$

\subsection{Parameter estimation}

The likelihood maximization based parameter estimation process involves two basic steps, the generation/evaluation of the candidate parameter values and the evaluation of the likelihood function. In the logit-based conventional discrete choice models, the likelihood function has a closed form, so the evaluation of likelihood, gradient, and hessian of the function is trivial. Gradient-based search methods like Newton-Raphson (NS), Broyden-Fletcher-Goldfarb-Shanno (BFGS), Berndt-HallHall-Hausman (BHHH), David-Fletcher-Powell, Polak-Ribiere conjugate gradient, and simulated annealing (Ben-Akiva and Lerman, 1985; Washington et al., 2003; and Train, 2009) are used to estimate the parameters and their statistical properties.

In the case of parameter estimation for probit models, the evaluation of the likelihood function becomes nontrivial because of the involvement of the multi-dimensional integral. In the case of the classic probit model, the multidimensional integral involved in the likelihood function is approximated using one of several methods: numerical integration, tabulation, numerical approximation, and Monte Carlo (MC) simulation (Sheffi et al., 1982). MC Simulation is most widely used when the likelihood function is evaluated using various simulation techniques like accept-reject (AR), smoothed AR, and Geweke-Hajivassiliou-Keane (GHK). The resulting approximate log-likelihood function is called a simulated loglikelihood (SLL). The gradient of the function required for an optimization problem can be approximated by dividing the change in SLL by the change in the parameter values (Train, 2009). Bolduc (1999) suggests a simulation-based procedure for the analytical solution of the gradient in the GHK-sim- 
ulator. Another approach for the probit model parameter estimation is the Bayesian-based Markov Chain Monte Carlo (MCMC) simulation technique that avoids the direct evaluation of the likelihood function. Instead it derives the posterior distributions from the prior belief and the data. The moments and other statistical properties are derived by sampling from the posterior distribution using simulation techniques like Metropolis-Hasting (M-H), adaptive M-H, and Gibb's sampler (Kim et al., 2002; Kim et al., 2003; and Train, 2009). Kim et al. (2003) used Markov chain Gibb's sampler to draw directly from posterior distribution and performed finite sample likelihood inference.

Bhat (2001) used a quasi-random MC simulation technique to estimate parameters for a mixed logit model. A Halton sequence for each dimension of the integral in the likelihood function was drawn by pairing $k$-sequences. The sequence ensured that the whole region under the integral is uniformly covered. The cyclic nature of the Halton sequence results in correlation issues. To avoid this problem, a scrambling technique was used, but this adds an exponential overhead with each dimension, so as to produce a "good" permutation (Hess and Polak, 2003). It is, however, not very clear what maximization criteria were used and how the approximate gradient/ scores and hessian were calculated. It is also not very clear how the local maxima were avoided in the estimation process.

Train (2009) outlined a Bayesian-based MCMC method for the parameter estimation in mixed logit models. Bayesian methods relax the constraint of maximizing the simulated-likelihood function, which could be complicated in cases in which there could be various local maxima and thus might result in identification problems. In Bayesian methods, the prior distribution plays an important role and is assumed to be near the values that globally maximize the likelihood function. Bayesian methods are also superior to standard simulated-likelihood maximization methods in terms of consistency and efficiency. In the case of large-scale demand model estimation, MC simulation, quasi-MC simulation, and MCMC simulation methods are commonly used (Bhat, 2001, Kim et al., 2002, Habib, 2009). Bhat (2005) and Habib and Miller (2009) used the quasi-random MC simulation procedure outlined in Bhat (2001) for the likelihood function that had extreme valued error terms and normally distributed parameters. Kim et al. (2002), von Haefen and Phaneuf (2004), and Habib (2009), used MCMC based on Metropolis-Hasting method to estimate their parameters from the likelihood function involving the normal distribution. The likelihood functions in the cases of von Haefen and Phaneuf (2004) and Habib (2009) had extreme valued error terms and normally distributed correlated parameters. Kim et al. (2002), on the other hand, had normally distributed correlated error terms as well. They used GHK simulator to evaluate the multidimensional integral involved within the log likelihood function. The statistical properties of the estimated parameters were computed using Gibb's sampling. The likelihood function in equation [11] also involves correlated error terms that are normally distributed. In the estimation of parameters from this function, we also decided to use Bayesian MCMC with Gibb's sampling approach. For the evaluation of the multidimensional normal probability function involved in equation [11], we used a technique based on randomized lattice rules that seeks to fill the hyper integration space evenly using a deterministic process. In principle, these lattice rules construct regular patterns, such that the projections of the integration points onto each axis produce an equidistant subdivision of the axis (Genz, and Bretz 2002, 2009). Robust integration error bounds are obtained by introducing additional shifts of the entire set of integration nodes in random directions. Since this additional randomization step is only performed to introduce a robust MC error bound, 10 simulation runs are usually sufficient. We preferred this method from the more widely used Halton sequence-based simulation procedure because it has been numerically proven to outperform Halton or Sobel sequences in terms of efficiency and doesn't suffer from correlation issues (Lai, 2009).

\subsection{Estimation procedure}

The procedure that we used to estimate parameters in equation [11] is as follows:

Let the parameters in the likelihood function be paired as $\zeta_{\mathrm{b}}=\left(\beta_{\mathrm{b}}^{\mathrm{r}}, \beta_{\mathrm{b}}^{\mathrm{c}}, \gamma, \rho\right)$, $\alpha$, and $\vartheta$. The parameters in $\zeta_{\mathrm{b}}$ with a mean of $\zeta_{b}$ are correlated to each other by matrix $\Omega_{\zeta}$.

$$
\begin{aligned}
& \text { 1. Initialize } \zeta_{b}, \alpha, 9, \bar{\zeta}_{b}, \Omega_{\zeta} \\
& \text { 2. Generate }\left\{\zeta_{b}, \mathrm{~b}=1, \ldots, \mathrm{B}\right\} \\
& \psi\left(\zeta_{b} \mid\left\{q_{b t}, t=1, \ldots, T\right\}, \alpha, \bar{\zeta}_{b}, \Omega_{\zeta}\right\} \propto \operatorname{det}\left|\Omega_{\zeta}\right|^{\frac{1}{2}} \exp \left[-\frac{1}{2}\left(\zeta_{b}-\bar{\zeta}_{b}\right)^{\prime} \Omega_{\zeta}^{-1}\left(\zeta_{b}-\bar{\zeta}_{b}\right)\right] \prod_{t} L_{b t}
\end{aligned}
$$

\section{Where}

$\psi$ is a $N \times 1$ vector representing all the alternatives $t$ represents the decision occasion

Generate a random number $\tau_{\psi} \rightarrow \mathrm{N}(0,0.0025)$, then the candidate value of $\zeta_{\mathrm{b}}$ for iteration $k$ will be:

$$
\zeta_{b}^{k}=\zeta_{b}^{(k-1)}+\tau_{\psi}
$$

Accept this new value with the probability:

$$
\min \left[\frac{\exp \left[-\frac{1}{2}\left(\zeta_{b}^{(k)}-\bar{\zeta}^{(k)}\right)^{\prime} \Omega_{\zeta}\left(\zeta_{b}^{(k)}-\bar{\zeta}^{(k)}\right)\right] \prod_{t}^{T} L_{b t}^{k}}{\exp \left[-\frac{1}{2}\left(\zeta_{b}^{(k-1)}-\bar{\zeta}^{(k-1)}\right)^{\prime} \Omega_{\zeta}\left(\zeta_{b}^{(k-1)}-\bar{\zeta}^{(k-1)}\right)\right] \prod_{t}^{T} L_{b t}^{k-1}}, 1\right]
$$


3. Generate $\zeta_{\mathrm{b}}$ from

$\psi\left(\bar{\zeta}_{b} \mid\left\{\zeta_{b}, b=1, \ldots, B\right\}, \Omega_{\zeta}\right\}=N\left(\frac{\sum_{1}^{B} \zeta_{b}}{B}, \frac{\Omega_{\zeta}}{B}\right)$

4. Generate $\Omega_{\zeta}$ from

$\psi\left(\Omega_{\zeta} \mid\left\{\zeta_{b}, b=1, \ldots, B\right\}, \bar{\zeta}_{b},\right\} \propto$ Inverted Wishart $\left(d_{0}+B . D_{0}+\sum_{1}^{B}\left(\zeta_{b}-\bar{\zeta}_{b}\right)^{\prime}\left(\zeta_{b}-\bar{\zeta}_{b}\right)\right)$

Where $\mathrm{d}_{0}$ is the prior degrees of freedom and $\mathrm{D}_{0}$ is the sum of squares of $\Omega_{\zeta}$

5. Generate $\alpha$ from

$\psi\left(\alpha \mid\left\{q_{b t}, b=1 \ldots B\right.\right.$ and $\left.\left.t=1, \ldots, T\right\},\left\{\zeta_{b}, b=1 \ldots B\right\}, \bar{\alpha}_{0}, \Sigma_{0}\right\}$

$\propto \operatorname{det}\left|\Omega_{0}\right|^{\frac{1}{2}} \exp \left[-\frac{1}{2}\left(\alpha-\bar{\alpha}_{0}\right)^{\prime} \Omega_{0}{ }^{-1}\left(\alpha-\bar{\alpha}_{0}\right)\right] \prod_{b}^{B} \prod_{t}^{T} L_{b t}$

$\alpha_{0}$ and $\Omega_{0}$ are the prior parameters

Generate a random number $\tau_{\alpha} \rightarrow \mathrm{N}(0,0.01)$, then the candidate value of $\alpha$ for iteration $k$ will be:

$$
\alpha^{\mathrm{k}}=\boldsymbol{\alpha}^{(\mathrm{k}-1)}+\tau_{\alpha}
$$

Accept this new value with the probability:

$\min \left[\frac{\exp \left[-\frac{1}{2}\left(\alpha^{k}-\bar{\alpha}_{0}\right)^{\prime} \Omega_{0}{ }^{-1}\left(\alpha^{k}-\bar{\alpha}_{0}\right)\right] \prod_{b}^{B} \prod_{t}^{T} L_{b t}^{k}}{\exp \left[-\frac{1}{2}\left(\alpha^{(k-1)}-\bar{\alpha}_{0}\right)^{\prime} \Omega_{0}{ }^{-1}\left(\alpha^{(k-1)}-\bar{\alpha}_{0}\right)\right] \prod_{b}^{B} \prod_{t}^{T} L_{b t}^{(k-1)}}, 1\right]$

\section{Generate 9 from}

$\psi\left(\vartheta \mid\left\{q_{b t}, b=1 \ldots B\right.\right.$ and $\left.\left.t=1, \ldots, T\right\},\left\{\zeta_{b}, b=1 \ldots B\right\}, \bar{\vartheta}_{0}, \Sigma_{0}\right\}$

$$
\propto \operatorname{det} \mid \Omega_{0^{\prime}}{ }^{\frac{1}{2}} \exp \left[-\frac{1}{2}\left(\vartheta-\bar{\vartheta}_{0}\right)^{\prime} \Omega_{0}^{-1}\left(\vartheta-\bar{\vartheta}_{0}\right)\right] \prod_{b}^{B} \prod_{t}^{T} L_{b t}
$$

$\vartheta_{0}$ and $\Omega_{0}$, are the prior parameters

Generate a random number $\tau_{9} \rightarrow \mathrm{N}(0,0.01)$, then the candidate value of $\vartheta$ for iteration $k$ will be:

$$
9^{\mathrm{k}}=9^{(\mathrm{k}-1)}+\tau_{9}
$$

Accept this new value with the probability:

$$
\min \left[\frac{\exp \left[-\frac{1}{2}\left(\vartheta^{k}-\bar{\vartheta}_{0}\right)^{\prime} \Omega_{0}{ }^{-1}\left(\vartheta^{k}-\bar{\vartheta}_{0}\right)\right] \prod_{b}^{B} \prod_{t}^{T} L_{b t}^{k}}{\exp \left[-\frac{1}{2}\left(\vartheta^{(k-1)}-\bar{\vartheta}_{0}\right)^{\prime} \Omega_{0}{ }^{-1}\left(\vartheta^{(k-1)}-\bar{\vartheta}_{0}\right)\right] \prod_{b}^{B} \prod_{t}^{T} L_{b t}^{(k-1)}}, 1\right]
$$

\section{Iterate back to step 1}

The simulation has to be run for a sufficient number of iterations before drawing inferences. It is suggested that around 25,000 iterations should be enough for the burn-in (Kim et al., 2002; von Haefen and Phaneuf, 2004; Train, 2009; and Habib, 2009). Gibb's sampling is then done to construct the distributional summary statistics for $\zeta b, \Omega_{\zeta}, \alpha, 9$. Gibb's sampling induces a serial correlation in the parameters. To avoid this correlation, it is also suggested that every 10th iteration be used in the simulation after warm up (von Haefen and Phaneuf, 2004; and Train, 2009).d in the simulation after warm up (von Haefen and Phaneuf, 2004; and Train, 2009).

\subsection{Solution identification}

Parameter estimation from the data based on the underlying model structure is fundamentally an optimization problem that may have a non-unique solution set. The identification problem is the problem of determining what conclusions drawn from the data about model parameters are feasible (Manski, 1995; Train, 2009). Walker et al. (2007) defined the identification problem as the problem of determining the set of restrictions to impose in order to obtain a unique vector of consistent parameter estimates. These restrictions can be on the range in which the parameter values should exist, acceptable goodness-of-fit values for the estimated model and definition of the regions in the search space in which to search. In this context, it seems that the Bayesian method based on MCMC simulation with Gibb's sampling does a better job of properly identifying the solution. Compared to the quasi-MC methods, the approach proposed in this paper, gives more flexibility in defining the search space and guiding the search in the proper direction. This is because the search is based on a prior distribution. The prior distribution corresponds to the knowledge that we already have about the parameters and their correlations. It is rarely the case that we do not have any idea about the sign and/or scale of the parameter values. We can thus control the starting direction of the search based on our prior beliefs about the solution. Secondly, the M-H-based search process is more controlled and directed. The $\mathrm{M}-\mathrm{H}$ search updates the value of the parameter distributions based on the increase in the likelihood from the new values. The statistical properties of the parameters in the solution are drawn from the posterior distribution of the parameters that are not just based on the likelihood values from the data, but also on the prior distribution and the search process. Lastly, by introduction of the Hicksian good, it is ensured that the solution space for the problem is reduced to finding only those parameters that represent the set of quantities within the technological constraints. The parameters estimation also takes into account the fact that a builder will not only invest in built space to the extent it can maximize profit but also has the option of investing elsewhere (or for that matter not investing at all). 


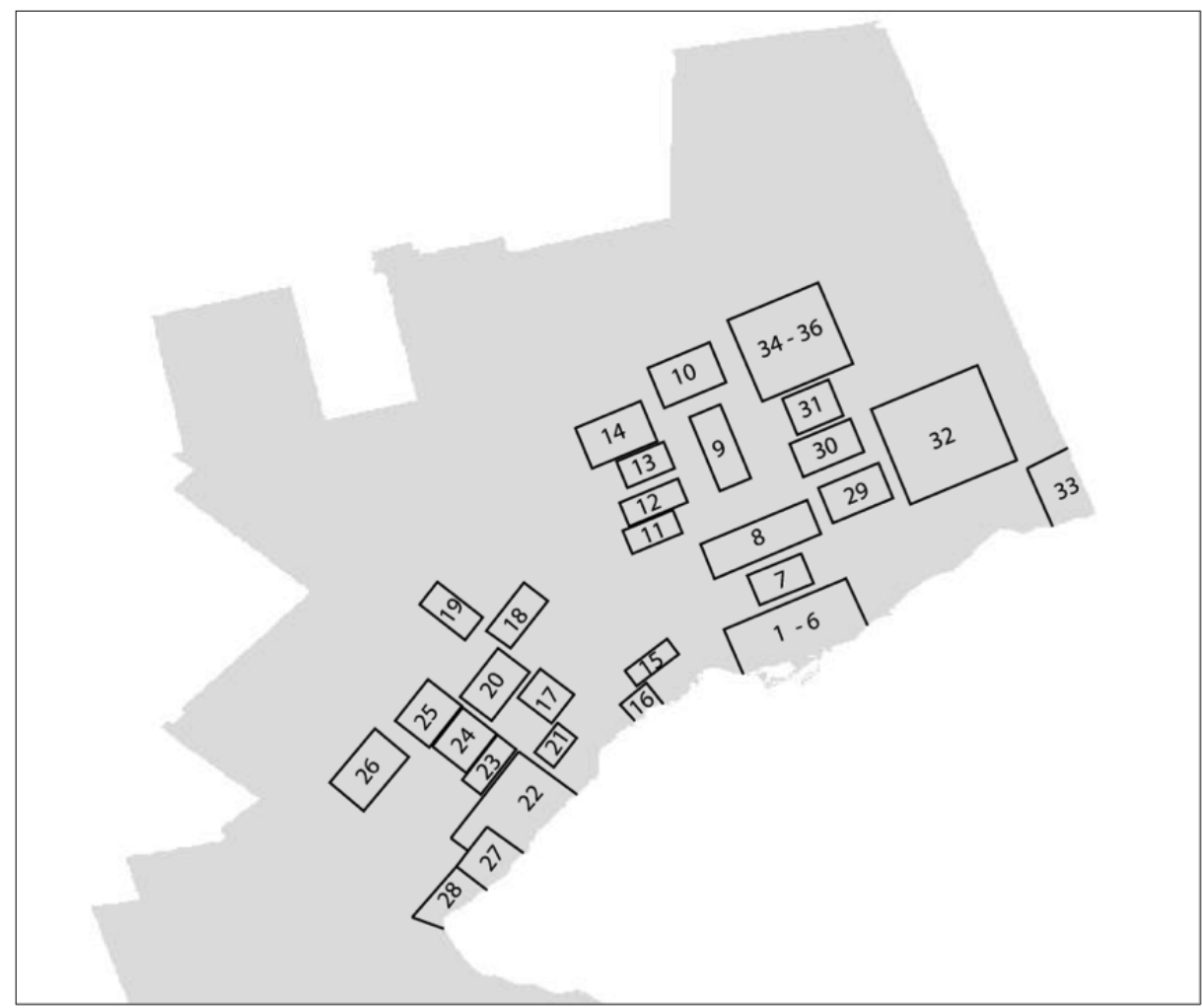

Figure 3: Study area and approximate location of the business nodes

Table 1: Summary statistics

\begin{tabular}{|c|c|c|c|c|c|}
\hline Variable & Description & Mean/Proportion & Std. Dev. & Min. & Max. \\
\hline \multicolumn{6}{|c|}{ Dependent Variable } \\
\hline Supply_A & Supply of Type A (1000 sq. ft.) & 76.57 & 226.78 & 0 & 2601.88 \\
\hline Supply_B & Supply of Type B (1000 sq. ft.) & 11.92 & 44.99 & 0 & 525.00 \\
\hline Supply_C & Supply of Type C (1000 sq. ft.) & 2.14 & 12.58 & 0 & 150 \\
\hline \multicolumn{6}{|c|}{ Independend Variable } \\
\hline Built_A & Already Built Type A in the Node (million sq. ft.) & 1.833 & 3.815 & 0 & 24.667 \\
\hline Built_B & Already Built Type B in the Node (million sq. ft.) & 1.307 & 1.448 & 0 & 5.987 \\
\hline Built_C & Already Built Type C in the Node (million sq. ft.) & 0.368 & 0.438 & 0 & 1.640 \\
\hline Gr_Rtl_Rt_A & Average Rent of Type A in the Node (CAN \$) & 25.46 & 6.888 & 2.30 & 50.30 \\
\hline Gr_Rtl_Rt_B & Average Rent of Type B in the Node (CAN \$) & 20.82 & 5.525 & 1.77 & 40.89 \\
\hline Gr_Rtl_Rt_C & Average Rent of Type C in the Node (CAN \$) & 18.73 & 5.000 & 7.40 & 37.62 \\
\hline Vac_Rt_A & Vacancy Rate of Type A in the Node & 0.139 & 0.077 & 0 & 37.62 \\
\hline Vac_Rt_B & Vacancy Rate of Type B in the Node & 0.160 & 0.096 & 0 & 0.56 \\
\hline Vac_Rt_C & Vacancy Rate of Type C in the Node & 0.158 & 0.175 & 0 & 1 \\
\hline Con_Wrks & Number of Construction Workers in the GTA (x1000) & 41.93 & 9.87 & 2.73 & 61.30 \\
\hline Wage_Rt & Hourly Wage Rate for Construction Workers & 18.27 & 2.69 & 12.83 & $22 / 91$ \\
\hline Con_Cost & Construction Cost per sq. ft. & 85.15 & 13.75 & 70.5 & 117.55 \\
\hline
\end{tabular}




\section{Application to office space supply}

Using the model formulation proposed in section 2, here we estimate a model for the new office space supply evolution in the GTA. The office space market in the GTA is a vibrant and growing market. The GTA is the third largest financial center in North America, after New York and Chicago. It is the center of activity in Canada for various office-based employment sectors, including finance, information technology, banking, insurance, and legal consulting. The consistent demand of high-quality office space with good accessibility and location arising from the office-based employment sectors has driven the growth in the office space market in the GTA. In addition to its relevance in understanding travel behavior, modeling and understanding the office space market in general and office space supply in particular have high economic benefits. The large capital requirements and long development periods make office investment riskier than other types of built space (Tse and Webb, 2003). Using office space models for forecasting and understanding the working of the building industry could decrease these investment risks. Few aggregate (country, municipality or central business district-suburban level) office space supply models can be found in the real estate and integrated land-use and transportation modeling literature. There are very few examples, however, of serious modeling efforts at the more disaggregate submarket level. This is in spite of the fact that there is strong evidence to suggest that submarkets within a metropolitan area are temporally asynchronous from each other in terms of growth and are characterized by a high level of agglomeration by industry type. The availability of certain types of office floor space has an effect on firm location and relocation decisions as well. Another important dimension in the modeling of the office space market is that the location, quality, and quantity are interconnected decisions. At anytime, a location may have excess stock of one type but is under stocked in another type. Similarly, some locations are suitable for only a few specific types of built space and not suited for others. For instance, downtown Toronto has a high concentration of Type A and B office space, but rarely Type $\mathrm{C}$ space (BOMA, 2009). The quantity that could be built at a certain location is also influenced by the neighborhood characteristics (zoning bylaws, technological constraints).

In the real estate literature, quantity is mostly modeled at a very high level of aggregation (Lentz and Tse, 1977; Rosen, 1984; Viezer, 1999; Hendershott et al., 1999; McDonald, 2000; Nanthakumaran et al., 2000; Tse and Webb, 2003; Ho, 2005; and Fürst, 2006). Operational integrated urban modeling frameworks model these decisions at a lower level of aggregation (census tracts, small grids) but do not treat them as related decisions within a single framework (Martínez, 1996; Martínez and Hurtubia, 2006; Martínez and Henríquez, 2007; Waddell et al., 2003, 2008; Waddell and Ulfarsson, 2003, 2004; Hunt and Abraham, 2003; and Miller et al., 2011). Instead the individual dimensions are modeled separately, and then some kind of simulation or rule-based allocation is used to simulate the built space evolution. In Integrated Land Use Transportation and Environment (ILUTE) for instance, Miller et al. (2011) used a separate model for the location choice probabilities for each type of dwelling and another model for the quantities to produce in the study area. These two models are then used in a MC simulation to allocate the new stock to individual locations.

The building industry generally, and in the case of the GTA in particular, is an oligopoly with very few firms, and these firms move in and out of the market very frequently with the boom and bust cycles of the built space market (Buzzelli and Harris, 2003). It is important to bring in the builders' behavior within the decision modeling framework. Their attitude toward risk taking and the expectation of profit might vary among individual builders. A modeling framework that can incorporate these issues is currently missing in the literature. Farooq et al. (2010) reported a high spatial variation in the rent of office space. It was also reported that there were not only inter-cluster variation but also intra-cluster variations. Under the profit maximizing assumption for the builder of space, this variation in rent will influence the decision to select the best location for the new office space. Considering the mentioned shortcomings in the existing literature, the estimation of a multidimensional decision model for office space that models the when, where, what, and how much decisions in a single framework is a significant contribution toward better understanding office space supply evolution.

We used the office space data collected by Coldwell Banker Richard Ellis (CBRE) consultants for the GTA from 1986 to 2005 to estimate the model. Office space is distributed across the study area in various identifiable clusters. Based on their geographic concentration in various regions, CBRE divided the study area into 36 visually identifiable business nodes (Figure 3 ) in the survey. The CBRE dataset that was used in this study is a quarterly account of the total inventory and market conditions, including new supply, vacancy rates, gross and net rent levels, and absorption rates in these business nodes for different types of office space. For the estimation of the model here, we converted the data to yearly values and used the data that were available from the first quarter of 1986 to the third quarter of 2005. The dataset classified office space into four standard types (A, B, C, and G), as defined by the Building Owners and Managers Association (BOMA). This is a subjective classifica- 
tion that uses a combination of factors including rent, building finishes, system standards and efficiency, building amenities, location/accessibility, and market perception (BOMA, 2009). Type A floor space has a high-quality standard finish, state-ofthe-art systems in the building, exceptional accessibility, and a definite market presence. Downtown Toronto and regional centers are dominated by Type A office space. Type A space has higher than average rents for the area. Type $\mathrm{B}$ office space has fair to good facilities and infrastructure, while Type $\mathrm{C}$ office space buildings only provide a functional space at a lower rent level compared to the area average. Type G office space is government-owned buildings. Type $\mathrm{G}$ buildings don't follow the general demand for office space in the market and are not included in this analysis. Table 1 provides the summary statistics and description of the dependent and explanatory variables used in the estimated model.

\subsection{Model estimates}

Using the dataset described in the previous section, we estimated the model of new office space supply for the GTA. The estimation process was implemented in $\mathrm{R}$ statistical language. This language was selected due to the open source nature of the language and availability of various support packages. The code is written in a very generic form and could be readily used for estimation of models based on the framework proposed in section 2 . The execution time for the estimation code is around six hours. The large computation time is due to the fact that we need to run the MCMC process for a long time to warm it up, and then to avoid correlation among iterations, we only use one in 10 iterations to generate distributions for the parameters. In the future we intend to work on a faster implementation of the algorithm.

The dependent variable here is the probability of selection of a vector $(3 \times 1)$ of quantities for each type of office space to be built in a business node $\mathrm{n}$ at certain year $\mathrm{t}$ from 1986 to 2005 . The explanatory variables used in the model represent the market conditions and land-use characteristics of the business node and the state of the regional economy at the time of the decision to build. Parameter estimates and associated statistics are reported in Table 2. Table 3 reports the correlations between different types of office space. The constant term for Type A space is the highest, followed by Type B. For Type $\mathrm{C}$ the constant term is negative. This suggests that builders in general prefer to build higher-quality space. The office employment sector in the GTA is dominated by financial, accounting, law, and technology firms that generate demand for high-quality office space. Higher constants for Type A and B seem to be the response of builders to this demand and higher profit margins.
Haider and Miller (2004) reported the phenomena of spatial inertia in the new housing supply of the GTA. We observe the same phenomena in the office space supply. The attractiveness, which is captured by the amount of office space that is already available (Built_*), is the highest in case of Type A, while it is lowest in the case of Type C. The rent per square foot of the type of office space at the time of decision was used as the indicator of market and growth of office-based employment. In general, there is a positive effect of the supply decisions with the higher rents, and this effect is highest in the case of Type $\mathrm{C}$ buildings. This result is unexpected, as one would expect that the higher-quality space would be more sensitive to the increase in rent. One reason for this might be that in general there is a higher temporal variation found in the rent of Type $\mathrm{C}$ office space. While in the case of Type $\mathrm{A}$ and $\mathrm{B}$, the variation is both in terms of time and space. We used average vacancy rate in the node at time of decision as another indicator of the demand for office space. The model reports negative sensitivity of the supply decisions to the increase in vacancy rates. This effect is highest in the case of Type A space. A higher project cost is associated with Type A space, and at the same time, the revenue (indicated by rents) from it is highest as well. This explains the higher sensitivity to vacant space in the case of Type A supply decisions. The number of construction workers in the labor force at the time of decision is used as the indicator of building inertia and state of the regional economy. A positive effect is found on supply decisions due to the increase in the number of construction workers. With an increase in the wage rates, the cost of the project increases and thus affects the new office space supply decision in a negative fashion. Similar behavior is evident in the case of an increase in construction cost. Unfortunately, due to the unavailability of data, we were unable to model the probability of selection due to difference in cost by type.

The greater than one theta variable that represents the behavior of the builders toward risk shows that they are risk takers. Builders overbuild in the boom of construction cycles anticipating future revenues. This fact is evident from the discussion in the data description section. We would like to introduce more detailed behavior of the builders and possible heterogeneity in the model by further parameterizing this variable using data on builders' characteristics. The translation parameters that make the corner solutions possible are almost the same for all three types. The standard errors are also very small. We also tried various starting values; these resulted in approximately the same values. It would be interesting to observe the effect of fixing the value of translation parameter to 100 and running the estimation again. This way, we would be able to control the bias that variable translation parameters may have introduced 
in the estimation process. The scale parameter seems to be in the acceptable range. The rho parameter that is associated with the parameterization for the Hicksian good is also in the right range. A limitation that arises due to the use of Bayesian-based MCMC estimation process is the inability to generate modellevel goodness-of-fit statistics. The goodness-of-fit test for these types of methods is an evolving research topic. An alternate approach to test the goodness of fit for this model could be to use simulation forecasting and compare the results with the observed data. Once new data for the years post 2005 are available, we plan to perform the simulation tests.

Table 2: Model parameter estimates

\begin{tabular}{|l|r|r|r|}
\hline Parameters & \multicolumn{1}{|l}{ Estimates } & \multicolumn{1}{l|}{ Std. Error } & \multicolumn{1}{l|}{ t-statistics } \\
\hline Explanatory Variable \\
\hline Const_A & 29.71 & 0.182 & 163.29 \\
\hline Const_B & 19.99 & 0.112 & 178.74 \\
\hline Const_C & -10.72 & 0.119 & -89.75 \\
\hline Built_A & 1.76 & 0.202 & 8.68 \\
\hline Built_B & 0.68 & 0.057 & 11.94 \\
\hline Built_C & 0.57 & 0.082 & 6.99 \\
\hline Gr_Rtl_Rt_A & 0.69 & 0.219 & 3.13 \\
\hline Gr_Rtl_Rt_B & 0.80 & 0.166 & 4.81 \\
\hline Gr_Rtl_Rt_C & 1.11 & 0.108 & 10.31 \\
\hline Vac_Rt_A & -2.44 & 0.140 & -17.44 \\
\hline Vac_Rt_B & -1.15 & 0.167 & -6.91 \\
\hline Vac_Rt_C & -0.37 & 0.236 & -1.58 \\
\hline Con_Wrks & 0.84 & 0.072 & 11.73 \\
\hline Wage_Rt & -1.32 & 0.116 & -11.37 \\
\hline Con_Cost & -0.64 & 0.135 & -4.73 \\
\hline Model structure parameters & & \\
\hline Gamma_A & 100.10 & 0.080 & 1246 \\
\hline Gamma_B & 100.06 & 0.088 & 1135 \\
\hline Gamma_C & 99.88 & 0.138 & 723.28 \\
\hline Rho & 4.49 & 0.108 & 41.64 \\
\hline Alpha & 0.66 & 0.173 & 1.107 \\
\hline Theta & 1.59 & 0.226 & 15.44 \\
\hline
\end{tabular}

Table 3: Correlation matrix between the error terms (significant with $95 \%$ confidence)

\begin{tabular}{|l|r|r|r|}
\hline \multirow{2}{*}{} & \multicolumn{3}{|c|}{ Type of Office Space } \\
\cline { 2 - 4 } & A & B & \multicolumn{1}{c|}{ C } \\
\hline A & 1 & 0.25 & -0.25 \\
\hline B & & 1 & -0.10 \\
\hline C & & & 1 \\
\hline
\end{tabular}

\section{$4 \quad$ Discussion and concluding remarks}

We presented a model for new office space supply for the GTA. The model is based on the novel multidimensional decision modeling framework for the supply of new built space. The modeling framework assumes that builders attempt to maximize expected profit. To our knowledge, this work is the first that models the where, when, how much, and what type of office space to supply in a single framework at a fairly disaggregate spatial zoning system. The estimated model is dynamic in the sense that it captures the lagged effects of market conditions on new supply. We observed the phenomena of spatial inertia in terms of location choices for different types of office space. The behavior of the builders in terms of risk is explicitly incorporated and estimated in the model. Builders are risk takers and tend to overbuild in the boom cycles. In the future, we intend to bring more detail into the model in terms of builder behavior and heterogeneity among builders. That will require a specialized survey of builders and their characteristics and observations about their decision-making behavior in the context of new built space supply. The changes in the construction project's expected cost on a builder's decisions are also modeled. Depending on the data availability, we intend to introduce a more detailed representation of costs and their variation by type and location in the model.

The use of MCMC simulation has a shortcoming in the sense that the model fitness statistics cannot be estimated. On the estimation side, we intend to assess the goodness of fit for the model using simulation and comparison of simulation results with the observed data. Unfortunately, data for the new built space supply post 2005 is not currently available. Another option for assessing the performance of the model would be to randomly pick some observations from the current dataset and not include them in the estimation process. We could then test the prediction power of the model on the excluded observations. This would provide some indication concerning how good the model is; however, the size of the dataset would be reduced further (currently 720 observations), which might affect the quality of the estimation process. In the future, we 
also intend to develop an estimation procedure for the model using a maximum likelihood method. Such a method would require at least developing procedures to evaluate the first and second order derivative of the likelihood function (equation [11]) discussed in section 2. The advantage of using maximum likelihood-based estimation is that we would be able to compute goodness-of-fit values from the estimation process. On the other hand, we would have to exercise extra caution so as to avoid getting stuck in the local maxima during the estimation process. Due to the higher degree from of the likelihood function and the problem's dimensionality, it is not very clear if the global maximum exists. In any case, we want to make sure that the solution that we find makes sense and is usable in planning and forecasting. For instance, if we were to set the parameters value to infinity (with appropriate signs), that would give us the maximum value for the likelihood function, but the resulting model estimates would be of no use to us in terms of giving insight and predictive power.

This model is part of our ongoing efforts toward operationalization of the office space market within ILUTE modeling framework, currently under development at the University of Toronto. In the general market-clearing framework of ILUTE, the asking rent model captures the role of accessibility, neighborhood characteristics, quality of space, and market conditions to determine the asking price at each simulation year. The model of the asking rent of office space at the building level has already been developed by Farooq et al. (2010). The models for demand of office space in the GTA by smalland medium-sized firms have also been developed by Elgar et al. (2009). With the available demand and supply, the asking rents are then to be used in the market-clearing module to match the space to the demander at a transaction rent that is endogenously determined. In the next simulation year, the lagged transaction rents then influence the builders' decisions of where, how much, and what type of office space to supply.

Note that the model estimated in section 3 is based on 36 business nodes that are spatially quasi-independent submarkets. While in the context of modeling the office space supply it makes sense to use this spatial system, for the implementation of the model in ILUTE, we will need another level of model that distributes the built space within a business node to the census tracts or dissemination areas that are marked as commercial by the zoning bylaws. This model can be a similar location choice model as the one estimated for the new housing supply by Haider (2003). This model will then we used in a fashion similar to how we used Haider's model for operationalizing housing supply in ILUTE v1.0 (Farooq, 2010).

\section{References}

Ben-Akiva, M., and S.R. Lerman. 1985. Discrete choice analysis: theory and application to travel demand, Cambridge, Massachusetts: The MIT Press

Bhat, C.R. 2001. Quasi-random maximization simulated likelihood estimation of the mixed multinomial logit model, Transportation Research (Part B), 35: 677-693

Bhat, C.R. 2005. A multiple discrete-continuous extreme value model: formulation and application to discretionary time-use decisions, Transportation Research (Part B), 39: 679-707

Bhat, C.R. 2008. The Multiple Discrete-Continuous Extreme Value (MDCEV) model: role of utility function parameters, identification considerations, and model extensions, Transportation Research (Part B), 42: 274-303

Bolduc, D. 1999, A practical technique to estimate multinomial probit models in transportation, Transportation Research (Part B), 33: 63-79

BOMA. 2009. Building Class Definitions, http://www.boma. org/resources/classifications/Pages/default.aspx Accessed July 13, 2009

Buzzelli, M., and R. Harris. 2003. Small is transient: Housebuilding firms in Ontario, Canada 1978-98, Housing Studies, 18(3): 369-386

De Sousa, C.A. 2002. Brownfield redevelopment in Toronto: an examination of past trends and future prospects, Land Use Policy, 19(4): 297-309

Elgar, I., B. Farooq, and E.J. Miller. 2009 Modeling location decisions of office firms: introducing anchor points and constructing choice sets into the model system, Transportation Research Record, 2133: 56-63

Farooq, B. 2010. Evolution of Urban Built Space: Markets and Decisions, Ph.D. Thesis, University of Toronto

Farooq, B., E.J. Miller, and M. Haider. 2010, Hedonic analysis of office space rent, Transportation Research Record, 2174: 118-127

Fürst, F. 2006. Predictable or Not? Forecasting Office Markets with a Simultaneous Equation Approach, MPRA Paper 5262, University Library of Munich, Germany

Genz, A., and F. Bretz. 2002. Methods for the computation of multivariate t-probabilities, Journal of Computational and Graphical Statistics, 11: 950-971

Genz, A., and F. Bretz. 2009. Computation of multivariate normal and tprobabilities, Lecture Notes in Statistics, 195

Habib, K.M., E.J. Miller, and K.W. Axhausen. 2008. Weekly rhythm in joint time expenditure for all at-home and outof-home activities: application of Kuhn-Tucker demand system model using multiweek travel diary data, Transpor- 
tation Research Record, 2054: 64-73

Habib, K.M., and E.J. Miller. 2009. Modelling activity generation: a utility-based model for activity-agenda formation, Transportmetrica, 5(1): 3-23

Habib, K.M. 2009. Investigating multiple activity participation and time-use decision by using a multivariate KuhnTucker demand system model, Transportation Letters: The International Journal of Transportation Research, 1: 257-269

Haider M. 2003. Spatio-temporal modelling of housing starts in the Greater Toronto Area, Ph.D. Thesis, University of Toronto

Haider, M., and E.J. Miller. 2004. Modelling location choices of housing builders in the Greater Toronto Area, Transportation Research Record, 1898: 148-156

Hendershott, P.H., C.M. Lizieri, and G.A. Matysiak. 1999. The workings of the London office market, Real Estate Economics, 27(2): 365-387

Hess, S., and J.W. Polak. 2003. The shuffled Halton sequence. Working Paper, Centre for Transport Studies, Imperial College London

Ho, K.W.O. 2005. Modeling the dynamics of the Hong Kong office market under economic structural change, Environment and Planning (Part B: Planning and Design), 32: $111-125$

Hunt, J.D., and J.E. Abraham. 2003. Design and application of the PECAS land use modelling system presented at The 8th Computers in Urban Planning and Urban Management Conference, Sendai, Japan, May 2003

Hunt, J.D., J.E. Abraham, D. De Silva, M. Zhong, J. Bridges, and J. Mysko. 2007. Microsimulating space development in Baltimore, 12th International Conference of the Hong Kong Society for Transportation Studies, December 2007

Jittorntrum, K. 1978. An implicit function theorem, Journal of Optimization Theory and Applications, 25(4): 575-577

Kim, J., G.M. Allenby, and P.E. Rossi. 2002. Modeling consumer demand for variety, Marketing Science, 21: 229-250

Kim, Y., T.Y. Kim, and E. Heo. 2003. Bayesian estimation of multinomial probit models of work trip choice, Transportation, 30: 351-365

Lai, Y. 2009. Intermediate rank lattice rules and applications to finance, Applied Numerical Mathematics, 59: 1-2

Lentz, G.H., and K.S.M. Tse. 1977. Supply adjustments to demand shocks in the commercial real estate market, Real Estate Economics, 27: 231-262

Manski, C.F. 1995. Identification problems in the social sciences, Cambridge, Massachusetts: Harvard University Press

Martínez, F. 1996. MUSSA: land use model for Santiago city, Transportation Research Record, 1552: 126-134

Martínez, F.J., and F. Hurtubia. 2006. Dynamic model for the simulation of equilibrium states in the land use market, Networks and Spatial Economics, 6: 55-73

Martínez, F.J., and R. Henríquez. 2007. A random bidding and supply land use model, Transportation Research (Part B), 41(6): $632-651$

Martínez, F.J., and J. Roy. 2004. A model for residential supply, The Annals of the Regional Science, 38(3): 500-531

McDonald, J.F. 2000. Rent, vacancy and equilibrium in real estate markets, Journal of Real Estate Practice and Education, 3(1): 55-69

Miller, E.J., B. Farooq, F. Chingcuanco, and D. Wang. 2011. Historical Validation of Integrated Transport-Land Use Model System, Transportation Research Record, 2 (2255): 91-99

Nanthakumaran, N., C. Watkins, and A. Orr. 2000. Understating property market dynamics: insights from modelling the supply-side adjustment mechanism, Environment and Planning (Part A), 32: 655-671

Pinjari, A.R., and C.R. Bhat. 2009. A Multiple Discrete-Continuous Nested Extreme Value (MDCNEV) model: formulation and application to non-worker activity time-use and timing behavior on weekdays, Transportation Research (Part B), doi:10.1016/j.trb.2009.08.001

Rosen, K.T. 1984., Towards a model of the office building sector, AREUEA Journal, 12: 261-269

Sheffi, Y., R. Hall, and C. Daganzo. 1982. On the estimation of the multinomial probit model, Transportation Research (Part A), 16A(5-6): 447-456

Sommerville, C.T. 2001. Permits, Starts, and Completions: Structural Relationships versus Real Options, Real Estate Economics, 29(1): 161-190

Train, K.E. 2009. Discrete choice methods with simulation, Second Edition, Cambridge, Massachusetts: Cambridge University Press

Tse, R.Y.C., and J.R. Webb. 2003. Models of office market dynamics, Urban Studies, 40(1): 71-89

Viezer, T.W. 1999. Econometric integration of real estate's space and capital markets, Journal of Real Estate Research, 18(33): 503-519 
von Haefen, R.H., and D.J. Phaneuf. 2004. "Kuhn-Tucker demand system approach to non-market valuation," Applications of Simulation Methods in Environmental and Resource Economics, eds. R. Scarpa and A. Alberini, London: Kluwer Academic Publishers

Waddell, P., and G.F. Ulfarsson. 2003. "Dynamic simulation of real estate development and land prices within an integrated land use and transportation model system," Presented at: The 82nd Annual Meeting of the Transportation Research Board, January 12-16, 2003, Washington, DC

Waddell, P., A. Borning, M. Noth, N. Freier, M. Becke, and G.F. Ulfarsson. 2003. "Microsimulation of urban development and location choices: design and implementation of UrbanSim," Networks and Spatial Economics, 3(1): 43-67

Waddell, P., and G.F. Ulfarsson. 2004. "Introduction to urban simulation: design and development of operational models," Handbook in Transport, Volume 5: Transport Geography and Spatial Systems, eds. Stopher, Button, Kingsley, Hensher, Pergamon Press, 203-236

Waddell, P., L. Wang, and X. Liu. 2008. "UrbanSim: an evolving planning support system for evolving communities," Planning Support Systems for Cities and Regions, edited by: Richard Brail, Cambridge, Massachusetts: Lincoln Institute for Land Policy, 103-138

Walker, J.L., M. Ben-Akiva, and D. Bolduc. 2007. "Identification of parameters in Normal Error Component LogitMixture (NECLM) models," Journal of Applied Econometrics, 22: 1095-1125

Washington, S.P., M.G. Karlaftis, and F.L. Mannering. 2003. Statistical and econometric methods for transportation data analysis, Washington, DC: Chapman and Hall/CRC 